\title{
Demonstration of Improved Charge Transfer in Graphene/Au Nanorods Plasmonic Hybrids Stabilized by Benzyl Thiol Linkers
}

\author{
Giuseppe Valerio Bianco, ${ }^{1}$ Maria Michela Giangregorio, ${ }^{1}$ Maria Losurdo, \\ Alberto Sacchetti, ${ }^{1}$ Pio Capezzuto, ${ }^{1,2}$ and Giovanni Bruno ${ }^{1}$ \\ ${ }^{1}$ Istituto di Nanotecnologia (NANOTEC), CNR, Via Orabona 4, 70126 Bari, Italy \\ ${ }^{2}$ Department of Chemistry, University of Bari, Via Orabona 4, 70126 Bari, Italy \\ Correspondence should be addressed to Giuseppe Valerio Bianco; giuseppevalerio.bianco@cnr.it
}

Received 10 September 2015; Revised 26 November 2015; Accepted 29 November 2015

Academic Editor: Ashok K. Sundramoorthy

Copyright (C) 2016 Giuseppe Valerio Bianco et al. This is an open access article distributed under the Creative Commons Attribution License, which permits unrestricted use, distribution, and reproduction in any medium, provided the original work is properly cited.

Hybrids based on graphene decorated with plasmonic gold $\mathrm{(Au}$ ) nanostructures are being investigated as possible materials combination to add to graphene functionalities of tunable plasmon resonance and enhanced absorption at selected wavelength in the visible-near-infrared region of the spectrum. Here, we report a solution drop-casting approach for fabricating stable hybrids based on chemical vapor deposition (CVD) graphene and Au nanorods, which are able to activate effective charge transfer from graphene. We demonstrate that CVD graphene functionalization by benzyl thiol (BZT) provides the linker to strong anchoring, via S-Au bonds, Au nanorods to graphene. Optical measurements by spectroscopic ellipsometry give evidence of the introduction of plasmon resonances at 1.85 and $2.25 \mathrm{eV}$ in the Au nanorods/BZT/graphene hybrids, which enable surface enhanced Raman scattering (SERS) detection. Furthermore, an effective electron transfer from graphene to Au nanorods, resulting in an enhancement of p-type doping of graphene with a consequent decrease of its sheet resistance, is probed by Raman spectroscopy and corroborated by electrical measurements.

\section{Introduction}

The synergic combination of the extraordinary mechanical, thermal, and electronic properties of graphene $(\mathrm{G})$ with the peculiar functionalities of metal [1], semiconductor [2], and oxide [3] nanostructures is an important field of research with promising technological perspectives. Among those hybrids, immobilization of stable plasmonic gold $(\mathrm{Au})$ nanostructures onto graphene is an approach adopted to develop photoactive electrodes for solar cells [4] and electrocatalysis [5], as well as fabricate plasmonic enhanced graphene-based optical switches and photodetectors operating from the near infrared to the visible $[1,6]$ and SERS (surface enhanced Raman scattering) sensors [7].

Several approaches have been exploited for fabricating plasmonic hybrids based on G/Au nanostructures. For instance, nanoscale $\mathrm{Au}$ arrays have been fabricated on graphene by electron beam lithography [1] and wet chemical synthesis from colloidal solutions containing Au nanoparticles with a defined plasmonic response [7]. While largely used spherical Au nanoparticles have localized surface plasmon resonance (SPR) mainly in the visible range and redshifting with the increase of radius from approximately $2.5 \mathrm{eV}$ down to $2.0 \mathrm{eV}$ [8], Au nanorods (AuNRs) enable more interesting functions due to their anisotropic shape, which introduces splitting of the plasmon resonance in the longitudinal (L-SPR, excitation along the major axis of the AuNRs) and transverse (T-SPR, excitation along the minor radial axis of the AuNRs) modes, with the former tunable in the near-infrared range and the latter in the visible range [9]. Such peculiar tunability of AuNRs plasmonic properties in combination with graphene has demonstrated great potential for the fabrication of high sensitive and flexible surface enhanced Raman scattering (SERS) sensors [10, 11] and high-performance NIR photodetectors [9]. Furthermore, Au nanoparticles coupled to graphene have been demonstrated to dope graphene by electron transfer [12]. In this context, while it has been largely assumed that Au nanostructures yield p-type doping 
of graphene due to the difference in the work functions of $\mathrm{Au}(5.1 \mathrm{eV})$ and graphene $(4.5 \mathrm{eV})$, we have recently demonstrated that the size and shape of Au nanostructures introduce changes in the $\mathrm{Au}$ work function that enables tailoring of graphene doping from p-type to n-type [12]. Therefore, it is important to develop scalable methods of fabrication of G/AuNRs hybrids. Nevertheless, reproducible immobilizing and stabilizing of AuNRs from colloidal solutions onto CVD graphene are still challenging. For this reason, the majority of work in literature decorates with metal nanoparticles graphene oxide [13] that uses various functional groups to anchor nanoparticles. Furthermore, capping agents, used to prevent AuNRs aggregation in solution, can inhibit the direct interaction of AuNRs with graphene.

In this work, we propose benzyl thiol (BZT) as linker for stabilizing the anchoring of colloidal AuNRs on the surface of graphene obtained by chemical vapor deposition (CVD). While BZT is linked via $\pi-\pi$ stacking to graphene, a strong covalent Au-S bond binds AuNRs to BZT as schematized in Figure 1. Peculiarity of this approach is the direct drop-casting of cetyltrimethylammonium bromide (CTAB) capped AuNRs on BZT functionalized graphene, without performing any additional step of AuNRs capping agent exchange reaction in solution. We demonstrate the effectiveness of BZT linkers in enhancing the stability of the AuNRs/BZT/G hybrid and in activating electron transfer processes between graphene and AuNRs. To probe this electron transfer, we combine optical analyses of spectroscopic ellipsometry, which detects the plasmon response of the G/BZT/AuNRs hybrids, Raman spectroscopy, and electrical measurements of sheet resistance, which detect variation of doping of graphene. Moreover, SERS signal provides insights into the interaction of both graphene and AuNRs with BZT molecules.

\section{Experimental}

The graphene used in this work was grown by CVD on $25 \mathrm{~mm}$ thick copper foils in a quartz tube CVD reactor at $1000^{\circ} \mathrm{C}$ (heating rate of $20^{\circ} \mathrm{min}^{-1}$ ) using $\mathrm{CH}_{4}$ and $\mathrm{H}_{2}$ as precursors $\left(\mathrm{CH}_{4}: \mathrm{H}_{2}=100: 50\right)$ at a pressure of 4 Torr [14]. Samples were then cooled at a rate of $\sim 2^{\circ} \mathrm{C} \mathrm{min}^{-1}$ in 1 Torr of $\mathrm{H}_{2}$. The graphene was transferred onto glass substrates by the thermal release tape method [15], etching the copper substrate in a water solution of ammonium persulphate $(0.1 \mathrm{M})$.

Graphene functionalization with BZT (Sigma-Aldrich, product code B25401) was carried out by dipping for $30 \mathrm{~min}$ graphene in a $1 \mathrm{M}$ solution of $\mathrm{BZT}$ in $\mathrm{CH}_{2} \mathrm{Cl}_{2}$ (SigmaAldrich, product code 650463) and subsequent rinsing with $\mathrm{CH}_{2} \mathrm{Cl}_{2}$. AuNRs were deposited on graphene by drop-casting $0.5 \mathrm{~mL}$ of a water solution of CTAB stabilized AuNRs (SigmaAldrich, product code $716855-25 \mathrm{ML}$, diluted to a concentration of $113 \mu \mathrm{g} / \mathrm{mL}$ ). After water evaporation, the samples were subjected to three cycles of water (type II) rinsing.

AuNRs with transversal and longitudinal sizes of $25 \mathrm{~nm}$ and $50 \mathrm{~nm}$, respectively, were used in our experiments (as shown in the image of Figure 1), with the L-SPR and T-SPR modes expected at $1.98-2.15 \mathrm{eV}$ and $2.23-2.45 \mathrm{eV}$, respectively [16].

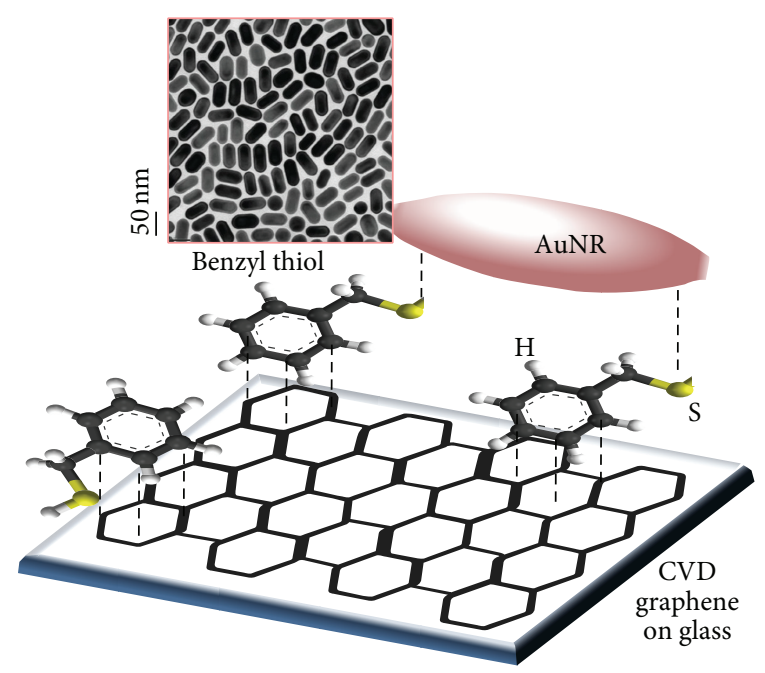

FIGURE 1: Schematic illustration of BZT linkers anchoring Au nanorods onto graphene. The $\pi-\pi$ interaction between BZT and graphene and the covalent S-Au bonds are indicated by dashed lines. The inset also shows the transmission electron microscopy image of the AuNRs.

Electrical measurements of sheet resistance, which give indication of graphene doping, were carried out using a fourpoint contact geometry in the van der Pauw configuration (MMR Technologies, Inc.) on a $5 \times 5 \mathrm{~mm}^{2}$ sampled area.

Raman spectra were collected using a LabRAM HR Horiba-Jobin Yvon spectrometer with the $532 \mathrm{~nm}$ and $633 \mathrm{~nm}$ excitation lasers to probe SERS effects in the AuNRs/G hybrids.

Ellipsometric spectra of the complex pseudodielectric function and extinction coefficient, $\langle k\rangle$, were acquired using a phase-modulated spectroscopic ellipsometer (UVISEL, Horiba-Jobin Yvon) in the $0.75-6.0 \mathrm{eV}$ spectral range with a resolution of $0.01 \mathrm{eV}$ to detect the plasmon resonance behavior of the AuNRs/G hybrids.

Atomic force microscopy (AFM, Thermomicroscope) was also used to probe the morphology of the hybrids.

\section{Results and Discussion}

Figure 2(a) shows the ellipsometric spectra of the pseudoextinction coefficient, $\langle k\rangle$, of CVD graphene supported on glass substrate before and after decoration with BZT and AuNRs. For comparison, the spectra of the AuNRs/BZT/G hybrid after three cycles of water immersion $(30 \mathrm{~min})$, rinsing, and drying, as well as the spectrum of the same AuNRs and of spherical Au nanoparticles with the same radial dimension of $25 \mathrm{~nm}$ directly on glass, are also shown. The $\langle k\rangle$ spectrum of the pristine graphene is dominated by an intense asymmetric peak around $4.8 \mathrm{eV}$, referred to as Van Hove peak, which mainly derives from the electron interband transitions at the M-point of the Brillouin zone [17]. Both graphene and glass being almost transparent in the energy range below $3.2 \mathrm{eV}$, the longitudinal (L-SPR) and transverse plasmon resonance (TSPR) modes of the AuNRs, respectively, at 1.85 and $2.25 \mathrm{eV}$ are clearly visible in the $\langle k\rangle$ spectra. Those localized SPR modes originate from the collective and coherent oscillations 


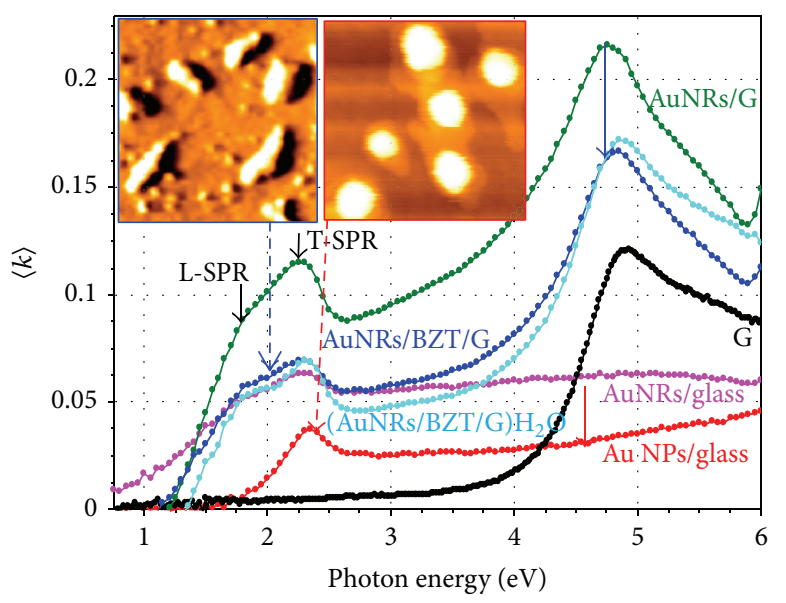

(a)

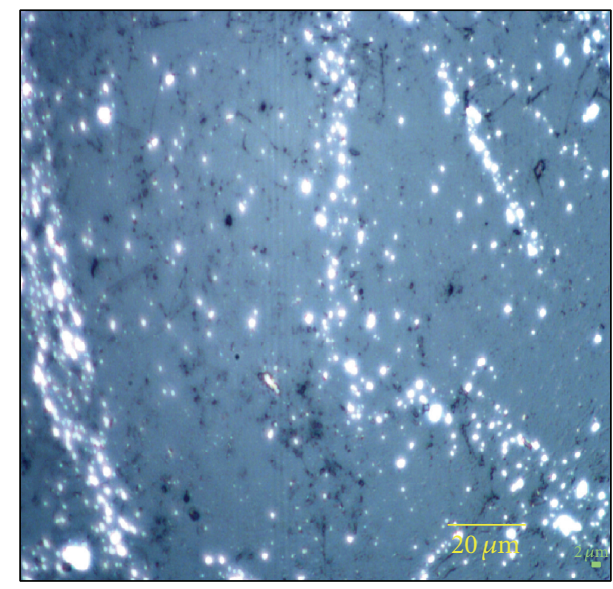

(c)

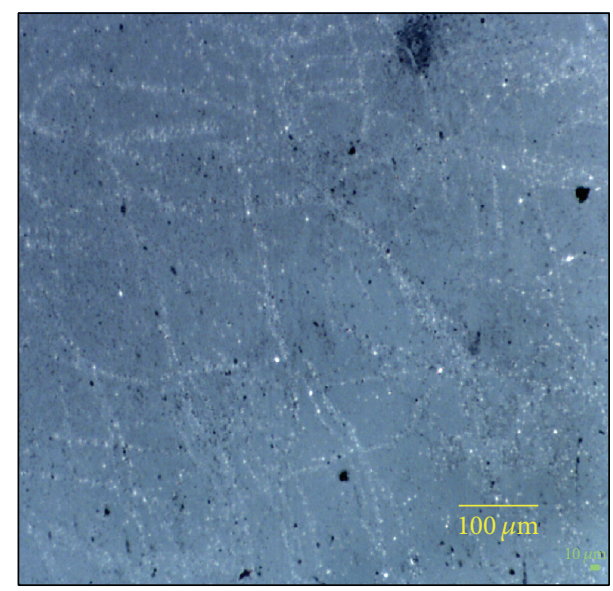

(e)

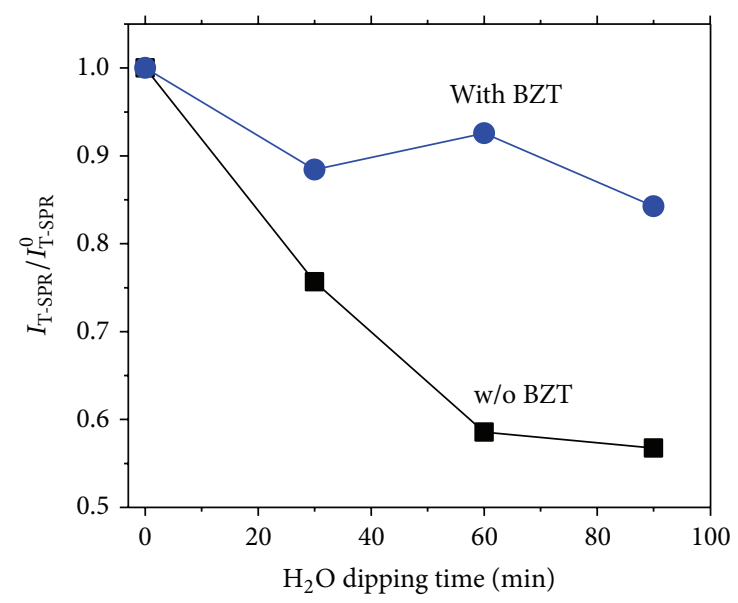

(b)

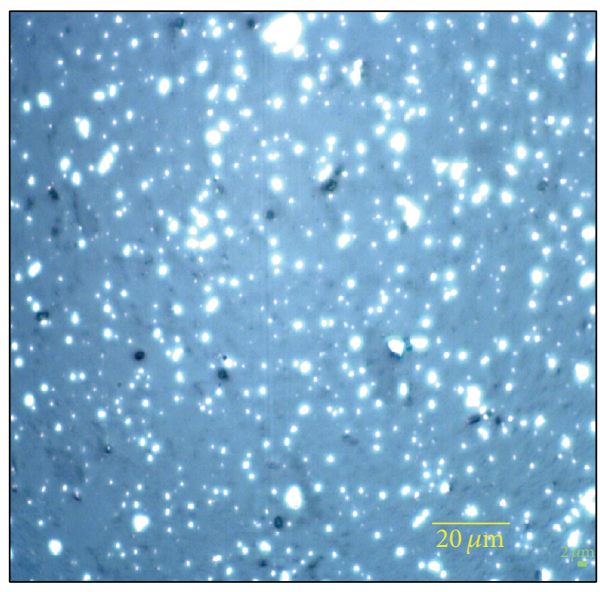

(d)

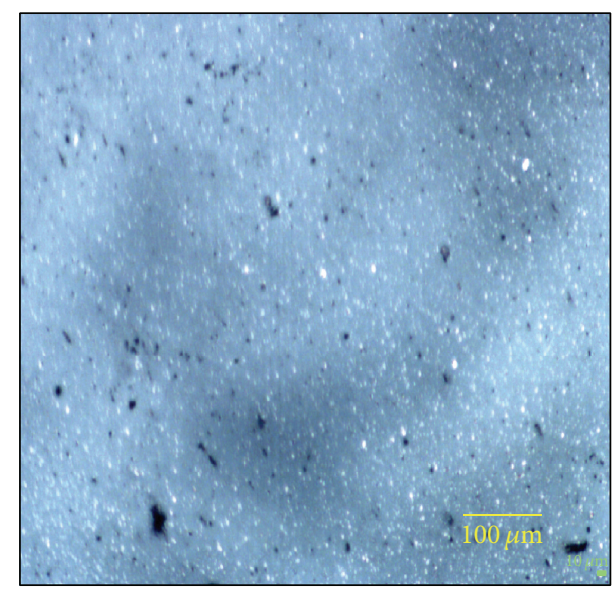

(f)

FIGURE 2: (a) Spectra of the pseudoextinction coefficient, $\langle k\rangle$, of CVD graphene supported on glass (black spectrum), of AuNRs directly drop-cast on graphene, and AuNRs/G (green spectrum), of the Au rods/BZT/G hybrid before (blue spectrum) and after (AuNRs/BZT/G) $\mathrm{H}_{2} \mathrm{O}$ (cyan spectrum) water rinsing. For comparison, the spectra of the AuNRs directly drop-cast on glass (and therefore with the capping CTAB) (magenta spectrum) and after thermal annealing to remove the CTAB capping (red spectrum) are also shown. (b) Evolution of the ratio between $I_{\mathrm{T}-\mathrm{SPR}}$ and $I_{\mathrm{T}-\mathrm{SPR}}^{0}$ (the latter being the intensity soon after drop-casting of AuNRs) upon three cycles of water immersion (30 min), rinsing, and drying. (c, d, e, f) Optical images of the surface morphologies of the (c, e) AuNRs/G and (d, f) AuNRs/BZT/G hybrids. The insets in (a) show the $400 \mathrm{~nm} \times 400 \mathrm{~nm}$ AFM images of AuNRs on BZT/G (left image) and of the annealed AuNRs/glass sample resulting in a change to spherical nanoparticles (right image). 
of the Au electron plasmon along the nanorod long and radial axes [18]. This assignment is supported by the comparison with the only SPR mode at $2.25 \mathrm{eV}$ of the spherical Au nanoparticles with the same radial size of $25 \mathrm{~nm}$ (see red spectrum of AuNPs/glass in Figure 2(a)).

The higher intensity of the spectrum of the AuNRs/G (AuNRs with the CTAB capping directly drop-cast on the graphene) is due to the inclusion of the CTAB, and the decreased spectrum of the AuNRs/BZT/G is because the BZT has been effective in displacing the CTAB. This can also be inferred by the comparison of the spectra of the AuNRs directly drop-cast on glass: in this case the CTAB has been removed by thermal annealing of the sample, so that a decrease of the spectrum amplitude by the CTAB removal is observed (see magenta and red spectra in Figure 2(a)). Noteworthy, the annealing also resulted in aggregation and melting of AuNRs that were transformed in spherical nanoparticles, as confirmed by the disappearance of the longitudinal SPR mode and appearance of only a SPR peak characteristic of spherical nanoparticles (see red spectrum of AuNPs/glass in Figure 2(a)). Furthermore, the $\langle k\rangle$ spectra of AuNRs/BZT/G hybrid before and after three cycles of water treatment are almost coincident, indicating that the AuNRs are stabilized through the BZT linker on the graphene. The slight observed variation in the amplitude of the spectra (compare blue and cyan spectra in Figure 2(a)) may be ascribed to the removal of some nanorods by solvation and/or to the removal of further CTAB residual from the sample. Figure 2(b) shows that when BZT is used as linker (blue points), the occurrence of such slight modification is limited to the first water immersion and rinsing cycle.

Conversely, when AuNRs are drop-cast directly on graphene, the ratio between $I_{\mathrm{T} \text {-SPR }}$ and $I_{\mathrm{T} \text {-SPR }}^{0}$ decreases to a value around 0.5 after three cycles of water treatment. Such decrease in the amplitude of the T-SPR of AuNRs could be related to a decrease of the surface density of the AuNRs due to their partial transfer into solution and/or aggregation upon solvation. In particular, the formation of AuNRs aggregates is a thermodynamically favored process whose driving force is the minimization of the high surface tension of isolated nanostructures.

In order to investigate the surface morphologies of the hybrids, we have taken advantage of the intense scattering in the visible of the plasmonic AuNRs to provide fast and direct information on their surface density and distribution on a large area by using a simple optical microscope. The optical photographs in Figures 2(c) and 2(d) confirm the presence of AuNRs aggregates (white spots in the pictures) of different sizes on both the AuNRs/G and AuNRs/BZT/G samples. However, without the BZT linker, the AuNRs aggregates are inhomogeneously distributed on the graphene (Figure 2(c)). The AFM topography in the inset of Figure 2(a) (left image) shows the presence of AuNRs with a height of $25 \mathrm{~nm}$, corresponding to the lower axis of the AuNR, and lateral sizes of approximately $100 \mathrm{~nm}$, which can be associated with a single AuNR and/or aggregates of two rods. Moreover, by further lowering the microscope optical magnification, it is possible to notice that the surface distribution of AuNRs directly drop-cast on graphene defines a patched morphology

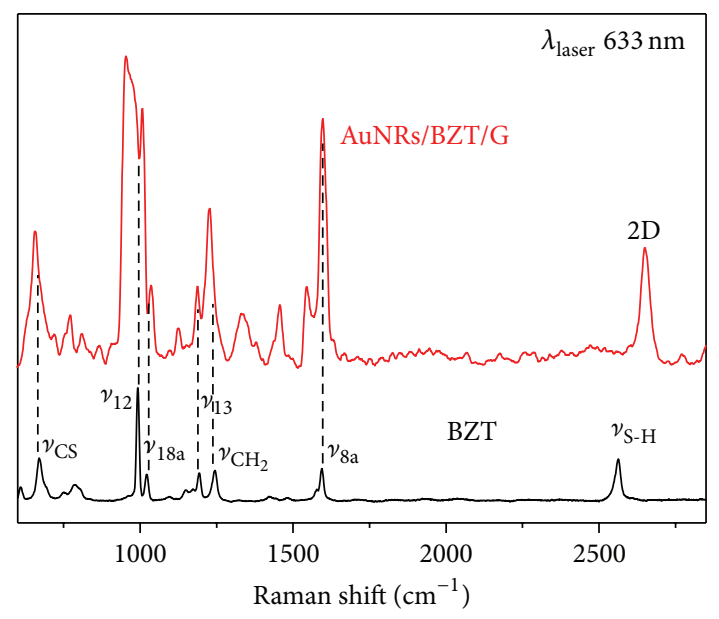

FIGURE 3: Raman spectrum of AuNRs/BZT/G acquired with a laser source operating at $633 \mathrm{~nm}$ (red spectrum). The reference Raman spectrum of BZT (black spectrum) is also reported for comparison.

(Figure 2(e)) that cannot be observed in the hybrid with BZT (Figure 2(f)). Although CVD graphene is a polycrystalline material, the sizes of such patches are not compatible with the grain sizes estimated for the CVD graphene produced in our laboratory [2]. The observed patched morphology is more consistent with the morphology of CVD graphene wrinkles [19], formed during graphene growth (at the Cu substrate step edges due to thermal stress) as well as during transferring to the glass substrate (especially for transferring of large area graphene samples) [19].

Figure 3 shows the Raman spectrum (red line) acquired for the AuNRs/BZT/G hybrid using a laser excitation source at $633 \mathrm{~nm}(1.96 \mathrm{eV}$, which is in the proximity of the L-SPR peak). For comparison, the reference Raman spectrum of BZT (black line) with the assignment of the most prominent Raman modes [20] is also shown. The graphene 2D Raman mode can be observed at $2660 \mathrm{~cm}^{-1}$. Below $1600 \mathrm{~cm}^{-1}$ the spectra are characterized by several peaks due to BZT, with the $v_{8 \mathrm{a}}$ mode of BZT overlapping the graphene G-peak at $1582 \mathrm{~cm}^{-1}$. The high intensity of the BZT Raman peaks is due to SERS Raman scattering signal enhanced by the AuNRs. In fact, these SERS peaks are minimized, and only the graphene Raman modes are visible, when a laser excitation source at $2.33 \mathrm{eV}(532 \mathrm{~nm})$, above the AuNRs plasmon resonance, is used (see blue spectrum in Figure 4(a)). The SERS enhancement is minimized using such laser energy because of the lower ratio of scattering to absorption from photons interacting with AuNRs [21], due to the higher contribution of the gold interband transitions absorption [22].

Furthermore, considering that SERS enhancement is a function of the distance between the probed molecule (BZT in this case) and the plasmonic antenna (AuNRs in this case) and that it decays for a distance higher than $1.2 \mathrm{~nm}$ [23] (i.e., less than half the length of a fully extended CTAB molecule), the presence of the BZT SERS modes in Figure 3 indicates the proximity of the BZT as well as of graphene to the AuNRs antenna, and hence that BZT has been effective in replacing the CTAB capping. Moreover, the absence of the $v_{\mathrm{SH}}$ peak in 


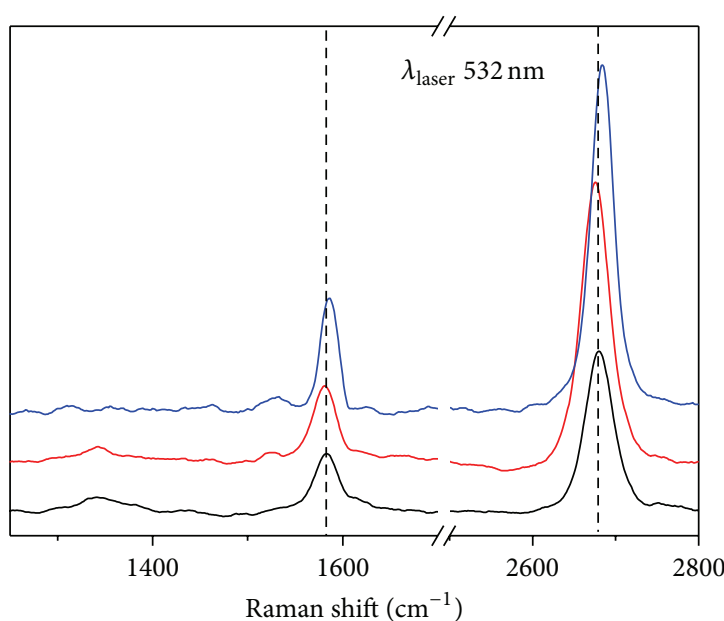

- Graphene

BZT/G
_ AuNRs/BZT/G

(a)

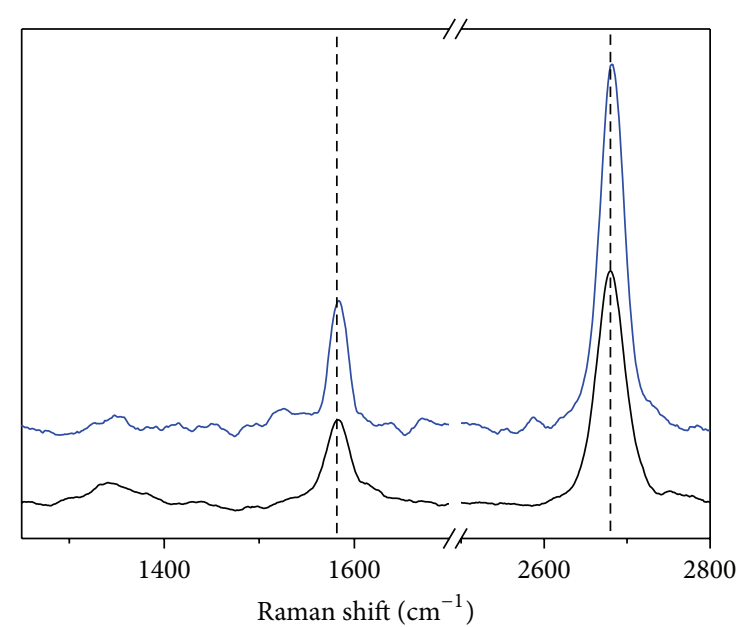

Graphene AuNRs/G

FIGURE 4: (a) Raman spectra of pristine graphene on glass (black spectrum), graphene functionalized with BZT (red spectrum), and the AuNRs/BZT/G hybrid. (b) Spectra of pristine graphene (black spectrum) and of the AuNRs/G hybrid (blue spectrum). Spectra have been acquired with the $532 \mathrm{~nm}$ laser source.

the SERS spectra demonstrates the covalent attachment of the BZT to the Au surface as thiolate by losing its proton.

The Raman analysis has also been exploited for investigating the effectiveness of charge transfer between graphene and AuNRs stabilized by BZT linkers. Specifically, the relative stiffening and softening of the G and 2D Raman modes are indicative of a change in the charge carrier concentration in graphene [24]. Such dependence on doping of the position of the graphene Raman modes has been reported for Raman analysis performed with laser sources operating at different energies [25] (including $1.96 \mathrm{eV}, 633 \mathrm{~nm}$ ). However, here, to minimize the contribution of the $v_{8 \mathrm{a}}$ SERS mode of BZT and to better estimate the graphene G-peak position, a laser source at $2.33 \mathrm{eV}(532 \mathrm{~nm})$ has been preferred. Figure 4(a) shows the Raman spectra of graphene as transferred onto the glass substrate (black line), after BZT drop-casting (red line), and after AuNRs anchoring (blue line). Upon BZT functionalization, a redshift of both the $G$ and $2 D$ peaks, from 1582 to $1579 \mathrm{~cm}^{-1}$ and from 2679 to $2676 \mathrm{~cm}^{-1}$, respectively, is measured, which is consistent with a decrease in the intrinsic p-doping characterizing CVD graphene [24]. This is also corroborated by an increase in the graphene sheet resistance from 1670 to $2500 \Omega /$ sq. The intrinsic p-doping of pristine CVD graphene mainly derives from the adsorption of water molecules or other contaminants from air, which act as hole-dopants [26]. Conversely, the doping effect of monosubstituted benzenes, such as the BZT, depends on the electron-donating or electron-withdrawing character of their substituents defined by their Hammett constant [27]. In particular, a negligible doping effect is expected for adsorbed BZT because of the low positive value (0.12) of its Hammett constant. Therefore, the observed changes in Raman modes and sheet resistance can be ascribed to the occurrence of a strong and stable $\pi-\pi$ interaction between graphene and BZT also able to displace the previously adsorbed air contaminants on graphene. Moreover, the stiffening of the $\mathrm{G}\left(1586 \mathrm{~cm}^{-1}\right)$ and $2 \mathrm{D}\left(2684 \mathrm{~cm}^{-1}\right)$ Raman modes in the spectra of the AuNRs/BZT/G hybrid (blue line) demonstrates that the anchoring of AuNRs by BZT also promotes electron transfer from graphene to AuNRs consistently with the work function of gold $(5.1 \mathrm{eV}$ is the theoretical reported value, while $4.75 \mathrm{eV}$ is the experimental value measured by us by Kelvin probe microscopy) [12] higher than that of graphene (4.5 eV) [12]. Thus, AuNRs act as p-dopants and lead to a decrease of the graphene sheet resistance (from 2500 to $850 \Omega / \mathrm{sq}$ ).

In contrast, when AuNRs are directly drop-cast on graphene, without the BZT, the G-peak in Raman spectrum remains stable at $1583 \mathrm{~cm}^{-1}$ (Figure $4(\mathrm{~b})$ ) and no changes in the sheet resistance are observed. This can be due to the fact that any direct interaction and charge transfer between the graphene and AuNRs are inhibited by the AuNRs capping agent (CTAB), by the spatial confinement of Au nanorods on graphene wrinkles as well as by the contaminants adsorbed on graphene.

\section{Conclusions}

BZT molecules are effective in stabilizing gold nanorods on graphene and in preventing their aggregation on the surface. The longitudinal and transverse plasmon resonance modes detected by spectroscopic ellipsometry together with the Van Hove singularity of graphene have demonstrated the formation of plasmonic AuNRs/BZT/G hybrids. The linker BZT anchors to graphene by $\pi-\pi$ stacking and it anchors gold nanorods by strong covalent $\mathrm{Au}-\mathrm{S}$ bonds as demonstrated by Raman analysis. Raman and van der Pauw measurements have also demonstrated an effective electron transfer from the 
$\mathrm{Au}$ nanorods to graphene, which is useful to lower the sheet resistance of graphene. Thus, BZT functionalization is an effective and simple methodology for engineering optical and transport properties of stable and large area graphene/metal nanoparticles plasmonic systems.

\section{Conflict of Interests}

The authors declare that there is no conflict of interests regarding the publication of this paper.

\section{Acknowledgments}

The authors acknowledge funding from the European Commission Seventh Framework Programme under Grant Agreement no. 314578 "MEM4WIN" and the National Laboratory Sens \& Micro LAB Project (POFESR 2007-2013, Code no. 15) funded by Apulia Region.

\section{References}

[1] Z. Fang, Y. Wang, Z. Liu et al., "Plasmon-induced doping of graphene," ACS Nano, vol. 6, no. 11, pp. 10222-10228, 2012.

[2] C. Ingrosso, G. V. Bianco, M. Corricelli et al., "Photoactive hybrid material based on pyrene functionalized $\mathrm{PbS}$ nanocrystals decorating CVD monolayer graphene," ACS Applied Materials \& Interfaces, vol. 7, no. 7, pp. 4151-4159, 2015.

[3] X. Meng, D. Geng, J. Liu, R. Li, and X. Sun, "Controllable synthesis of graphene-based titanium dioxide nanocomposites by atomic layer deposition," Nanotechnology, vol. 22, no. 16, Article ID 165602, 2011.

[4] H. Hoshi, S. Tanaka, and T. Miyoshi, "Pt-graphene electrodes for dye-sensitized solar cells," Materials Science and Engineering B: Advanced Functional Solid-State Materials, vol. 190, pp. 47-51, 2014.

[5] W. Hong, H. Bai, Y. Xu, Z. Yao, Z. Gu, and G. Shi, "Preparation of gold nanoparticle/graphene composites with controlled weight contents and their application in biosensors," The Journal of Physical Chemistry C, vol. 114, no. 4, pp. 1822-1826, 2010.

[6] Q. Bao, K. P. Loh, G. Eda, and M. Chhowalla, "Graphene photonics, plasmonics, and broadband optoelectronic devices," ACS Nano, vol. 6, pp. 3677-3694, 2012.

[7] Y. Zhang, T. R. Nayak, H. Hong, and W. Cai, "Graphene: a versatile nanoplatform for biomedical applications," Nanoscale, vol. 4, pp. 3833-3842, 2012.

[8] S. K. Ghosh and T. Pal, "Interparticle coupling effect on the surface plasmon resonance of gold nanoparticles: from theory to applications," Chemical Review, vol. 107, pp. 4797-4862, 2007.

[9] Z. Xia, P. Li, Y. Wang, T. Song, Q. Zhang, and B. Sun, "Solutionprocessed gold nanorods integrated with graphene for nearinfrared photodetection via hot carrier injection," ACS Applied Materials \& Interfaces, vol. 7, no. 43, pp. 24136-24141, 2015.

[10] R. S. Norman, J. W. Stone, A. Gole, C. J. Murphy, and T. L. Sabo-Attwood, "Targeted photothermal lysis of the Pathogenic bacteria, Pseudomonas aeruginosa, with gold nanorods," Nano Letters, vol. 8, no. 1, pp. 302-306, 2008.

[11] T. H. D. Nguyen, Z. Zhang, A. Mustapha, H. Li, and M. Lin, "Use of graphene and gold nanorods as substrates for the detection of pesticides by surface enhanced raman spectroscopy," Journal of Agricultural and Food Chemistry, vol. 62, no. 43, pp. 1044510451, 2014.

[12] M. M. Giangregorio, W. Jiao, G. V. Bianco et al., "Insights into the effects of metal nanostructuring and oxidation on the work function and charge transfer of metal/graphene hybrids," Nanoscale, vol. 7, no. 30, pp. 12868-12877, 2015.

[13] J. Liu, S. Fu, B. Yuan, Y. Li, and Z. Deng, "Toward a universal 'Adhesive Nanosheet' for the assembly of multiple nanoparticles based on a protein-induced reduction/decoration of graphene oxide," Journal of American Chemical Society, vol. 32, pp. 72797281, 2010.

[14] M. Losurdo, M. M. Giangregorio, P. Capezzuto, and G. Bruno, "Ellipsometry as a real-time optical tool for monitoring and understanding graphene growth on metals," The Journal of Physical Chemistry C, vol. 115, no. 44, pp. 21804-21812, 2011.

[15] J. D. Caldwell, T. J. Anderson, J. C. Culbertson et al., “Technique for the dry transfer of epitaxial graphene onto arbitrary substrates," ACS Nano, vol. 4, no. 2, pp. 1108-1114, 2010.

[16] K. Moth-Poulsen, T. Jain, J. K. Sørensen, and T. Bjørnholm, "Electronics and self-assembly with single molecules," Material Matters, vol. 4, no. 3, pp. 80-82, 2009.

[17] V. G. Kravets, A. N. Grigorenko, R. R. Nair et al., "Spectroscopic ellipsometry of graphene and an exciton-shifted van Hove peak in absorption," Physical Review B, vol. 81, no. 15, Article ID 155413, 2010.

[18] S. A. Maier, Plasmonics: Fundamentals and Applications, Springer, 2007.

[19] V. E. Calado, G. F. Schneider, A. M. M. G. Theulings, C. Dekker, and L. M. K. Vandersypen, "Formation and control of wrinkles in graphene by the wedging transfer method," Applied Physics Letters, vol. 101, no. 10, Article ID 103116, 2012.

[20] C. A. Szafranski, W. Tanner, P. E. Laibinis, and R. L. Garrell, "Surface-enhanced raman spectroscopy of aromatic thiols and disulfides on gold electrodes," Langmuir, vol. 14, no. 13, pp. 3570-3579, 1998.

[21] W. Lee, S. Y. Lee, R. M. Briber, and O. Rabin, "Self-assembled SERS substrates with tunable surface plasmon resonances," Advanced Functional Materials, vol. 21, no. 18, pp. 3424-3429, 2011.

[22] M. Losurdo, M. M. Giangregorio, G. V. Bianco et al., "Size dependence of the dielectric function of silicon-supported plasmonic gold nanoparticles," Physical Review B-Condensed Matter and Materials Physics, vol. 82, no. 15, Article ID 155451, 2010.

[23] K. A. Willets and R. P. Van Duyne, "Localized surface plasmon resonance spectroscopy and sensing," Annual Review of Physical Chemistry, vol. 58, pp. 267-297, 2007.

[24] A. Das, S. Pisana, B. Chakraborty et al., "Monitoring dopants by Raman scattering in an electrochemically top-gated graphene transistor," Nature Nanotechnology, vol. 3, no. 4, pp. 210-215, 2008.

[25] M. Bruna, A. K. Ott, M. Ijäs, D. Yoon, U. Sassi, and A. C. Ferrari, "Doping dependence of the Raman spectrum of defected graphene," ACS Nano, vol. 8, no. 7, pp. 7432-7441, 2014.

[26] G. V. Bianco, M. Losurdo, M. M. Giangregorio, P. Capezzuto, and G. Bruno, "Exploring and rationalising effective n-doping of large area CVD-graphene by $\mathrm{NH}_{3}$," Physical Chemistry Chemical Physics, vol. 16, no. 8, pp. 3632-3639, 2014.

[27] B. Das, R. Voggu, C. S. Rout, and C. N. R. Rao, "Changes in the electronic structure and properties of graphene induced by molecular charge-transfer," Chemical Communications, no. 41, pp. 5155-5157, 2008. 

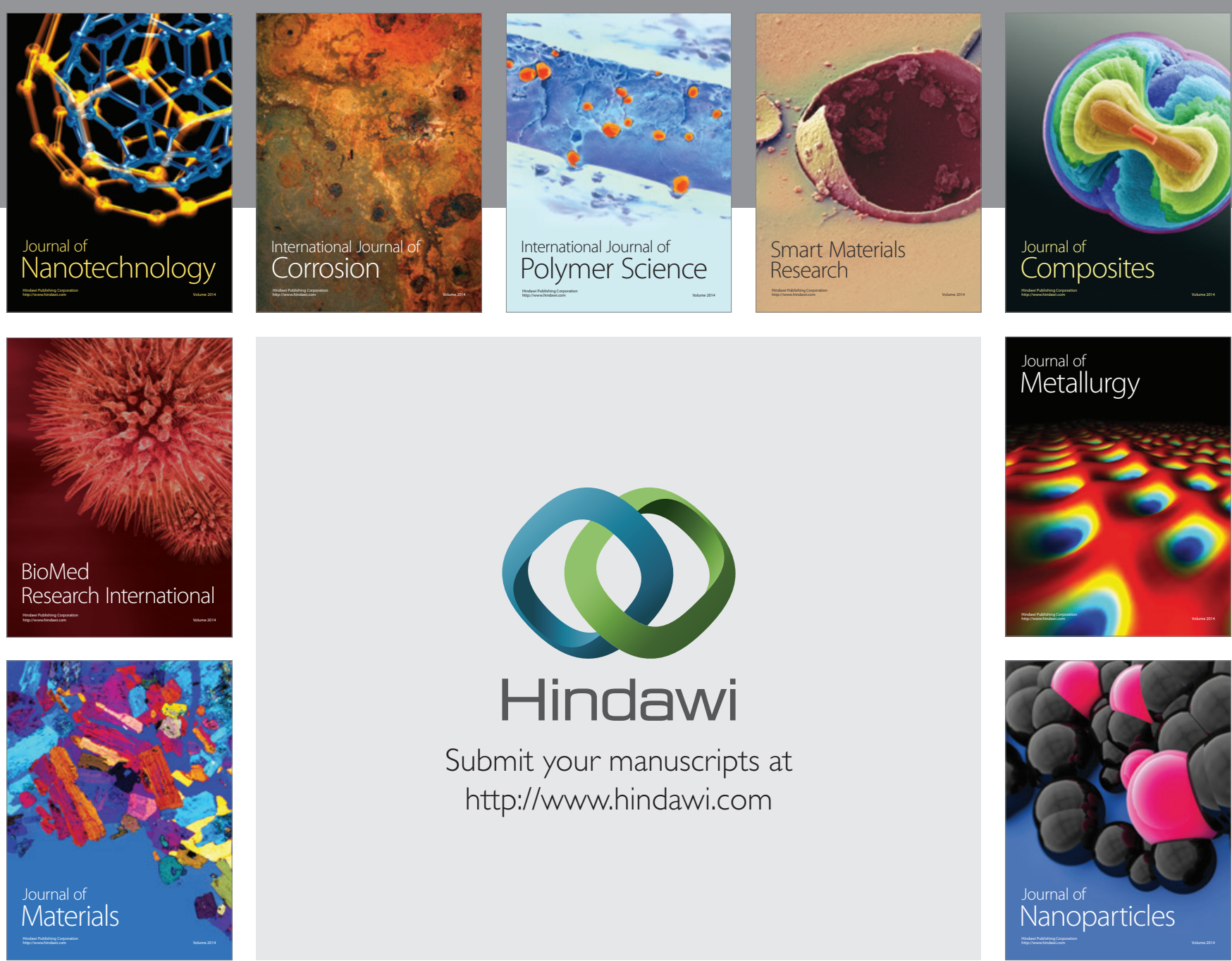

\section{Hindawi}

Submit your manuscripts at

http://www.hindawi.com

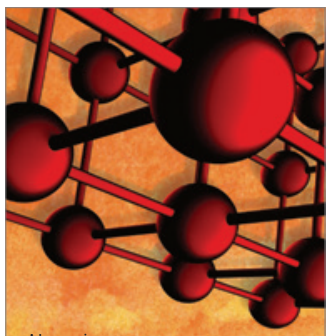

Materials Science and Engineering
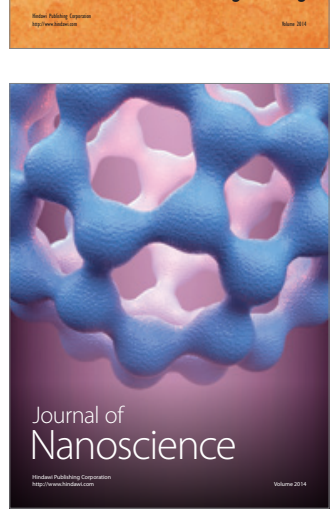
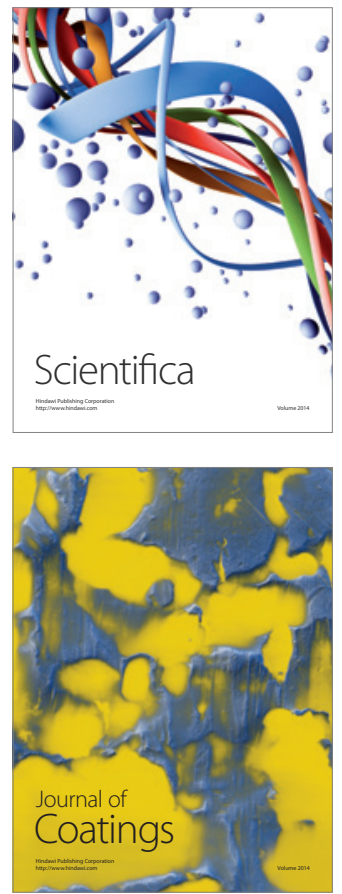
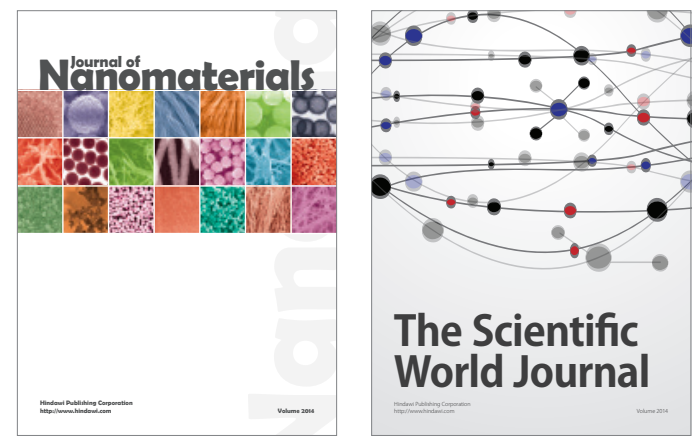

The Scientific World Journal
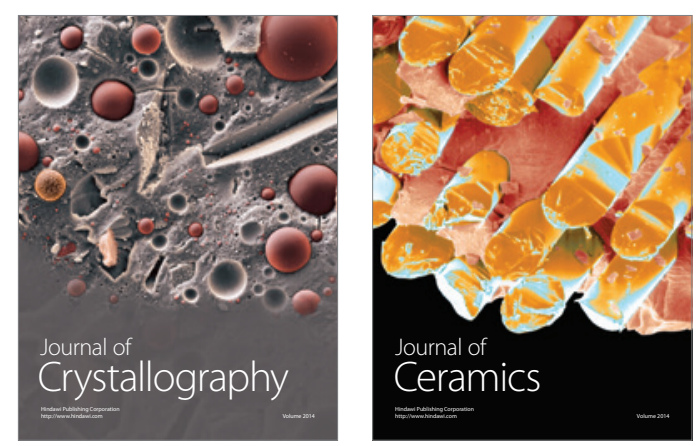
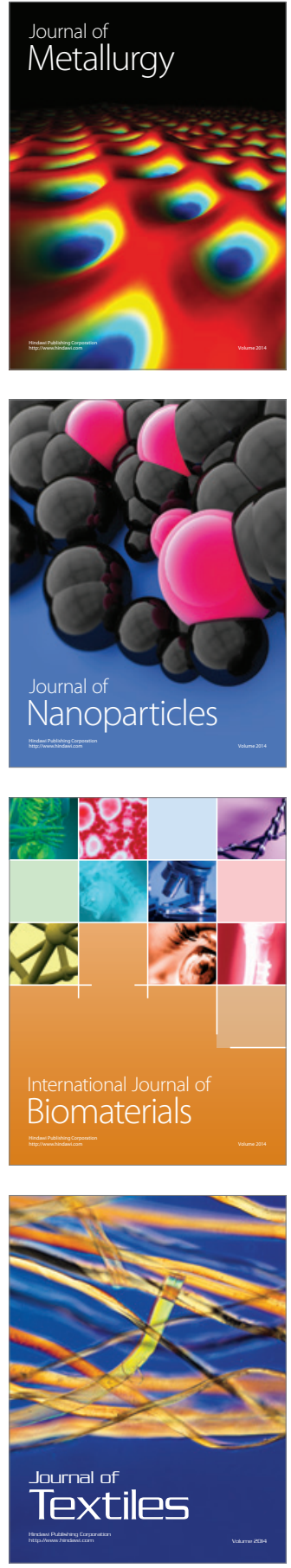